\title{
Functional Control of Network Dynamics Using Designed Laplacian Spectra
}

\author{
Aden Forrow, ${ }^{1, *}$ Francis G. Woodhouse, ${ }^{2, \dagger}$ and Jörn Dunkel ${ }^{1, \uparrow}$ \\ ${ }^{1}$ Department of Mathematics, Massachusetts Institute of Technology, \\ 77 Massachusetts Avenue, Cambridge, Massachusetts 02139-4307, USA \\ ${ }^{2}$ Department of Applied Mathematics and Theoretical Physics, Centre for Mathematical Sciences, \\ University of Cambridge, Wilberforce Road, Cambridge CB3 OWA, United Kingdom
}

(Received 5 January 2018; revised manuscript received 17 September 2018; published 7 December 2018)

Complex real-world phenomena across a wide range of scales, from aviation and Internet traffic to signal propagation in electronic and gene regulatory circuits, can be efficiently described through dynamic network models. In many such systems, the spectrum of the underlying graph Laplacian plays a key role in controlling the matter or information flow. Spectral graph theory has traditionally prioritized analyzing unweighted networks with specified adjacency properties. Here, we introduce a complementary framework, providing a mathematically rigorous weighted graph construction that exactly realizes any desired spectrum. We illustrate the broad applicability of this approach by showing how designer spectra can be used to control the dynamics of various archetypal physical systems. Specifically, we demonstrate that a strategically placed gap induces generalized chimera states in Kuramoto-type oscillator networks, tunes or suppresses pattern formation in a generic Swift-Hohenberg model, and leads to persistent localization in a discrete Gross-Pitaevskii quantum network. Our approach can be generalized to design continuous band gaps through periodic extensions of finite networks.

DOI: 10.1103/PhysRevX.8.041043

\section{INTRODUCTION}

Spectral band gaps control the behavior of physical systems in areas as diverse as topological insulators $[1,2]$, phononic crystals [3], superconductors [4], acoustic metamaterials [5], and active matter [6]. In addition to ubiquitous physical network models [7-10] ranging from aviation [11] to electronics [12], there is also considerable interest in virtual or computational networks [13] with fewer physical constraints, such as those recently used to create spiral-wave chimeras in coupled chemical oscillators [14]. Often, dynamics in such systems depend on the graph Laplacian [15,16] and in particular on its spectrum of eigenvalues. Traditionally studied in periodic lattice graph models $[3,5,6,17]$ and more recently also in hyperuniform systems [18], the targeted design of spectra

\footnotetext{
*aforrow@mit.edu

Present address: Mathematical Institute, University of Oxford, Andrew Wiles Building, Radcliffe Observatory Quarter, Woodstock Road, Oxford OX2 6GG, United Kingdom.

\$dunkel@mit.edu

Published by the American Physical Society under the terms of the Creative Commons Attribution 4.0 International license. Further distribution of this work must maintain attribution to the author(s) and the published article's title, journal citation, and DOI.
}

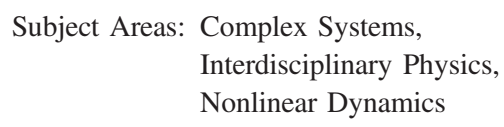

of any desired shape remains a major challenge in modern materials science [5,19]. Recent breakthroughs in 3D printing [20-23] and lithography [24] make it possible now to produce and explore network structures that go beyond the traditionally considered periodic lattice geometries.

Building on such experimental and theoretical progress, we present here a mathematically rigorous solution to the longstanding question of how any desired spectrum can be realized exactly on a suitably designed positively weighted network. Physical motivations necessitate variable edge weights: couplings in realistic networks, whether stiffnesses in spring systems or friendships in social networks, are not identical, and the common assumption of uniform weights is usually just a convenient simplification. Our construction of networks with specified eigenvalues allows us to place arbitrary gaps in the spectrum of the network Laplacian $L=D-A$, where $D$ and $A$ are the weighted degree and adjacency matrices, respectively. These gaps, finite analogs to band gaps in continuous systems, enable precise control over the dynamics in a wide range of graph-based physical systems. To follow the analogy, we will name an eigenvaluefree region in our finite networks that is comparable to the range of eigenvalues a discrete band gap (DBG). In a strict sense, band gaps can only exist in an extended system with continuous energy bands, and so we also show how to create continuous band gaps by tiling our DBG construction periodically (Sec. V). Designing a suitably weighted network 
topology in this way presents an alternative to control procedures based on adjusting model parameters or initial conditions on a given network [25]. The spectral approach towards functional control of network dynamics proposed here can, e.g., be directly implemented with recently developed computer-coupled oscillator setups [14] and may find future applications in networked optical lattices [26-29] and superconducting waveguide resonators [30].

After motivating of the general problem from a broader physics perspective (Sec. II), we will summarize and explain the main mathematical result (Sec. III). Subsequently, we focus on demonstrating its broad applicability explicitly for classical and quantum systems, by showing how suitably placed DBGs can induce chimera states $[31,32]$ in oscillator networks, control structural growth in pattern formation models, and facilitate state localization in quantum networks (Sec. IV). In parallel, we illustrate how our exact spectral construction can be combined with sparsification algorithms $[33,34]$ to yield simplified networks preserving DBGs. This approach complements the more traditional procedure of constructing graph ensembles with predefined statistical adjacency characteristics [35-38]. Finally, we discuss periodic extensions of finite networks as a systematic procedure for designing continuous band gaps (Sec. V).

\section{GRAPH LAPLACIANS}

A weighted simple graph $G$ is defined by its vertex set $V$, edge set $E$ containing unordered pairs of distinct vertices $(i, j)$, and corresponding edge weights $w_{i j}$. We consider the case with real, non-negative weights $w_{i j} \geq 0$ and set $w_{i j}=0$ if there is no edge between $i$ and $j$. The Laplacian of $G$ is the matrix whose off-diagonal elements are the negatives of the edge weights and whose diagonal elements are the weighted vertex degrees. That is, $L_{i j}=-w_{i j}$ for $i \neq j$ and $L_{j j}=\sum_{i \neq j} w_{i j}$.

The Laplacian matrix occurs naturally in a wide range of physical systems. Up to a sign, it is the discrete analog of the continuous Laplacian: where $\nabla^{2}$ appears in continuous models, $-L$ typically appears in the discrete version of the model. For example, the ubiquitous nearest-neighbor finite difference approximation to $\nabla^{2}$ arises as the graph Laplacian of a square lattice [39].

The simplest physical examples of network Laplacians come from spring systems and discrete random walks. If a set of identical masses moving in one dimension are coupled by springs with stiffness $w_{i j}$ between masses $i$ and $j$, the force on mass $i$ is exactly $-\sum_{j} L_{i j} x_{j}=$ $\sum_{j} w_{i j}\left(x_{j}-x_{i}\right)$. Here, $x_{i}$ is the coordinate of the $i$ th mass. The mechanics then decouple into $n$ oscillation modes corresponding to the eigenvectors of $L$, with the eigenvalues as squared frequencies.

Similarly, if a particle follows a random walk on a network, traveling from node $j$ to node $i$ with rate $w_{i j}$ (so the probability flow from $j$ to $i$ is the probability $p_{j}$ of being in state $j$ times the rate), then the probability distribution evolves in time according to

$$
\frac{d p_{i}}{d t}=-\sum_{j \neq i} w_{j i} p_{i}+\sum_{j \neq i} w_{i j} p_{j}=-\sum_{j} L_{i j} p_{j}
$$

The solution again comes from the eigendecomposition of $L$ : the eigenvalues determine the diffusion rate and the rate of decay to the stationary distribution.

It is natural, then, to ask whether we can control these eigenvalues. By designing the network appropriately, what spectra can we construct? There are two clear constraints. First, the rows and columns of $L$ sum to zero, implying that 1, the vector of all ones, is an eigenvector with eigenvalue zero. This corresponds to the stationary distribution for the random walk and rigid translation for the spring network. Moreover, the remaining eigenvalues must be non-negative by the Gershgorin circle theorem [40]. We will see in the next section that these are the only two restrictions for weighted networks.

\section{NETWORK CONSTRUCTION AND SPARSIFICATION}

The problem of recovering a network from its eigenvalues has been studied extensively, both from an algorithmic [41-43] and mathematical [44,45] perspective. However, with a few limited exceptions [45], most prior work has focused only on unweighted networks [46], where there are a finite number of graphs on $n$ vertices and thus only a finite number of possible spectra. A weighted graph with no weight constraints can be constructed to have any desired set of eigenvalues and eigenvectors [47], but demanding positive weights makes the construction more challenging. Previous research [47] has shown how to control the largest and smallest nonzero eigenvalues with some uncertainty. Here, we give an exact spectral construction.

\section{A. Spectral graph construction}

Our main result is that, given a set $\left\{\lambda_{i}\right\}$ of desired eigenvalues ordered so $\lambda_{1} \geq \ldots \geq \lambda_{n-1} \geq \lambda_{n}=0$, there is a weighted graph $G$ on $n$ vertices with non-negative edge weights whose Laplacian $L$ has spectrum $\lambda_{1}, \ldots, \lambda_{n-1}, 0$. We show this by explicitly constructing such a network. While for arbitrary spectra our construction may be one of multiple possible solutions for $G$, in some cases the underlying unweighted topology is necessarily a complete graph, as discussed below.

The Laplacian, which determines the graph, can be reconstructed from its eigenvalues and eigenvectors with the eigenvalue decomposition; we therefore need to find a set of eigenvectors that together with $\left\{\lambda_{i}\right\}$ give a graph 
Laplacian. In fact, the same set of eigenvectors $v^{(k)}$, $k=1, \ldots, n-1$, given by

$$
v_{i}^{(k)}= \begin{cases}\frac{1}{\sqrt{k(k+1)}} & i<k+1 \\ -\frac{k}{\sqrt{k(k+1)}} & i=k+1 \\ 0 & i>k+1\end{cases}
$$

suffices for any spectrum. These eigenvectors are strongly localized: for large $k$, only component $k+1$ has a value far from zero. The inverse participation ratio (four-norm) $\left\|v^{(k)}\right\|_{4}^{4}=1-2 k^{-1}+O\left(k^{-2}\right)$ indicates near-perfect localization $\left\|v^{(k)}\right\|_{4}^{4} \rightarrow 1$ for almost all $k$, itself a desirable phenomenon [16,48] which we will exploit later in the context of pattern formation. As the $v^{(k)}$ are mutually orthonormal and orthogonal to the vector of all ones, the matrix $L=\sum_{k=1}^{n-1} \lambda_{k} v^{(k)} v^{(k) \top}$ has the desired spectrum with $(1 / \sqrt{n}) \mathbf{1}$ as the final eigenvector for $k=n$ with eigenvalue zero. Constructing the Laplacian from the eigenvalue decomposition in this way guarantees that $L$ is symmetric. By explicitly computing the sum over $k$ for $i<j$, we find

$$
L_{i j}=\sum_{k=1}^{n-1} \lambda_{k} v_{i}^{(k)} v_{j}^{(k)} \leq-\frac{\lambda_{j-1}}{n},
$$

that is, that the off-diagonal elements of $L$ are all nonpositive (Appendix B); $L$ therefore corresponds to a graph with non-negative weight $-L_{i j}$ between vertices $i$ and $j$. If all of the eigenvalues are nonzero, all of the off-diagonal elements of $L$ will be nonzero and the resulting graph will be complete.

Some spectra can only be realized on complete graphs. A graph $G$ with approximately constant spectrum must be complete: if $L$ has nonzero eigenvalues $\lambda_{k}=\lambda+\epsilon_{k}$ for $k<n$ and $\lambda_{n}=0$, then

$$
\lambda I-L=\frac{\lambda}{n} \mathbf{1 1}^{\top}-\sum_{k=1}^{n-1} \epsilon_{k} v^{(k)} v^{(k) \top} .
$$

The off-diagonal elements of $\lambda I-L$, which equal the original edge weights of $G$, are therefore $(\lambda / n)+O(\epsilon)$. For small $\epsilon_{k}$, every edge has nonzero weight; if $\epsilon=0$, we have that the complete graph with equal weights is the only network with a fully degenerate spectrum. More commonly, the network that realizes a spectrum is not unique. Suppose, for instance that there are $k$ zero eigenvalues. The graph must have $k$ disconnected components. The set of eigenvalues of each component considered individually will be a subset of the eigenvalues of the entire graph: the components' eigenvalues form a partition of the full spectrum. Any partition where each group contains one zero can be realized by constructing the components as described above. If there is more than one nonzero eigenvalue and $k>1$, there will be multiple partitions that give nonisomorphic graphs; typically, there will be an enormous number of isospectral disconnected graphs.

For connected graphs, with only one zero eigenvalue, our construction also shows that the spectrum of any noncomplete weighted graph cannot uniquely specify that graph, in line with older results on, e.g., the spectra of trees [44]. Given any connected graph with at least one missing edge, our design can match the spectrum with a complete graph that cannot be isomorphic to the given one.

This construction allows us to create networks with precisely specified gaps. For instance, choosing $\lambda_{1}=$ $\lambda_{2}=\ldots=\lambda_{n / 2-1}$ and $\lambda_{n / 2}=\lambda_{(n / 2)+1}=\ldots=\lambda_{n-1}$ leads to a graph with edge weights $-L_{i j}=\lambda_{n-1} / n$ if $i>n / 2$ or $j>n / 2$ and $-L_{i j}=\left(2 \lambda_{1}-\lambda_{n-1}\right) / n$ otherwise (Appendix A); that is, there are two groups of vertices, one strongly connected within itself and one weakly connected to everything. Adding a small amount of noise to each eigenvalue then lifts the eigenvalue degeneracy while preserving the connectivity structure and retaining a gap [Figs. 1(b) and 1(c)].

\section{B. Sparsification}

Since complete graphs can be difficult to realize physically, we explore the effect of the sparsification-byresistances algorithm developed by Spielman and Srivastava [33]. Given an accuracy parameter $\epsilon$, this sparsification creates a network with $O\left((n \log n) / \epsilon^{2}\right)$ edges whose eigenvalues match the eigenvalues of the original network to within a multiplicative factor $1 \pm \epsilon$ with high probability. Sparsification by resistances aims to preserve the entire spectrum, not just a gap; future sparsification algorithms directly constructed to preserve a gap could therefore improve on its efficiency. In other applications, the networks of interest are virtual ones [14] and sparsification may not be necessary.

Rather than removing edges from the initial graph, the sparsification algorithm constructs a new graph starting from disconnected vertices. Specifically, we first compute the effective resistance $R_{i j}$ between every pair of vertices in the initial graph, treating the edge weights as conductivities. We then sample $q=(n \log n) / \epsilon^{2}$ edges at random, with the probability $p_{i j}$ of sampling edge $(i, j)$ proportional to $w_{i j} R_{i j}$. Each edge we sample is added to the sparsified graph with weight $w_{i j} /\left(q p_{i j}\right)$; if an edge is added multiple times, the weights are summed. Clearly, the new graph will have at most $q$ edges. We leave the argument that this preserves the spectrum to Ref. [33].

We can use the $1 \pm \epsilon$ multiplicative error bound to estimate the size of a discrete band gap after sparsification. Suppose we start from a network with eigenvalues $\lambda_{1}, \lambda_{2}$, and 0 , with some multiplicities, where $\lambda_{1}>\lambda_{2}$. The eigenvalues $\left\{\mu_{i}\right\}$ of the sparsified graph corresponding to $\lambda_{1}$ should be no smaller than $\mu_{i} \geq(1-\epsilon) \lambda_{1}$, while the 
(a)

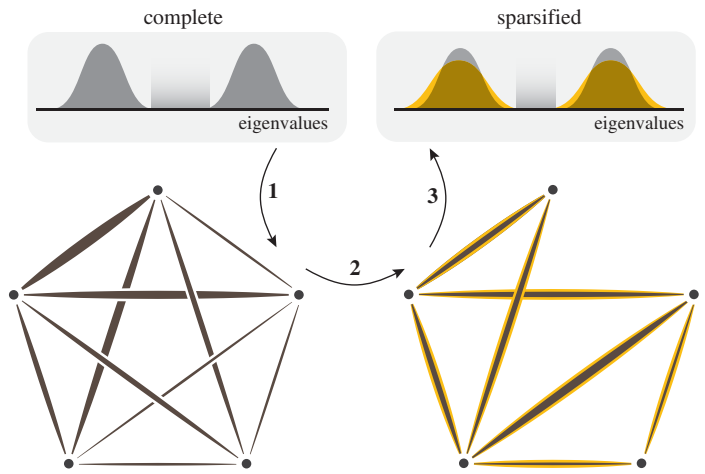

(c)

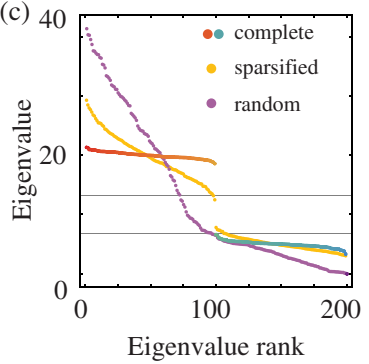

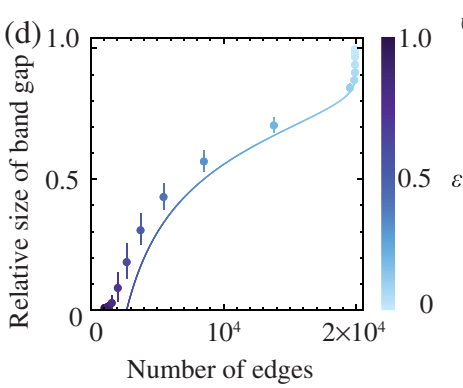

(b)

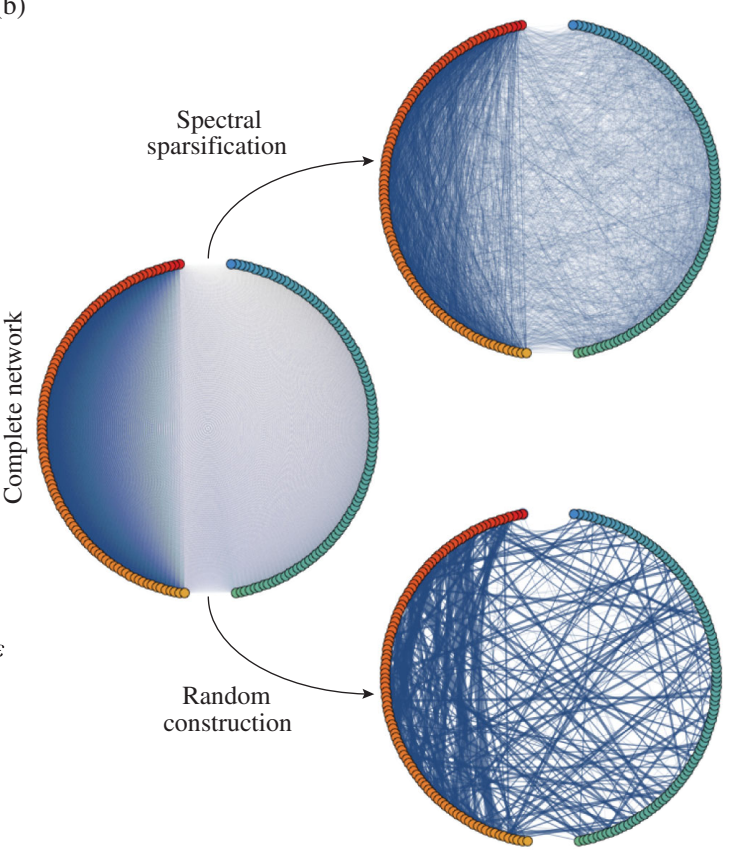

FIG. 1. Designing networks from spectra. (a) Schematic of DBG network construction. Given a spectrum of eigenvalues distributed in two (or more) groups, we build a graph with non-negative edge weights that realizes this spectrum exactly (1). Sparsification of this complete DBG network with the Spielman-Srivastava [33] algorithm (2) yields a new network with wider eigenvalue distributions and a smaller gap (3). (b) Example graphs used in applications below: Starting from a DBG graph on 200 vertices with 100 eigenvalues set to independent and identically distributed (IID) $\mathcal{N}(5,0.25)$ and 99 set to IID $\mathcal{N}(20,0.25)$ (left), sparsification with $\epsilon=0.5$ creates a new graph (top) with the number of edges reduced from 19900 to 3758. As a control, we also compare to a gapless random graph (bottom) with 362 edges and the same weighted vertex degrees as the original DBG graph (Appendix C). (c) The eigenvalues for the graphs in (b). The mode on the complete DBG network with the $k$ th largest nonzero eigenvalue is supported on the first $k+1$ vertices, counted counterclockwise from the top red vertex, and highly localized on vertex $k+1$, which is colored to match in (b). Gray lines indicate the borders of the unstable region for the Swift-Hohenberg model with the parameters used in Fig. 3. (d) Sparsified networks retain a significant gap even for relatively large $\epsilon$. Each point shows the mean number of edges and gap size at fixed $\epsilon$ between 1 (left) and 0.01 (right), starting from a graph on 200 vertices designed to have $100 \times$ eigenvalue 5 and $99 \times$ eigenvalue 20 . The solid curve shows the worst-case gap estimate, reduction by a factor of $1-\frac{5}{3} \epsilon$. Sample size is 1000 for $\epsilon \geq 0.1$ and 300 for $\epsilon<0.1$. Error bars are \pm 1 standard deviation; horizontal error bars are smaller than the marker size.

eigenvalues $\left\{\nu_{i}\right\}$ corresponding to $\lambda_{2}$ should be no larger than $\nu_{i} \leq(1+\epsilon) \lambda_{2}$. The sparsified graph should therefore have a gap $\Delta=\min _{i} \mu_{i}-\max _{i} \nu_{i}$ of size

$$
\Delta \geq\left(1-\frac{\lambda_{1}+\lambda_{2}}{\lambda_{1}-\lambda_{2}} \epsilon\right)\left(\lambda_{1}-\lambda_{2}\right)
$$

That is, the gap contracts by a factor at most $1-\left[\left(\lambda_{1}+\lambda_{2}\right) /\left(\lambda_{1}-\lambda_{2}\right)\right] \epsilon$. For the parameters used in Fig. 1(d), this is $\left(1-\frac{5}{3} \epsilon\right)$.

\section{APPLICATIONS}

We now demonstrate the practical potential of DBGs with three generic network models. In each case, we compare the dynamics on a complete DBG network [Fig. 1(b), left] both to a sparsified approximate DBG network [Fig. 1(b), top] and to a random connected network [Fig. 1(b), bottom] constructed to have the same weighted vertex degrees as the
DBG network (Appendix C). The gap is approximately preserved in the sparsified network and vanishes entirely in the random graph [Figs. 1(c) and 1(d)]. Matching the degrees in the random graph to the DBG network ensures that any differences in dynamics are not due to differences in coarse features like the average connectivity, but rather are likely caused by the spectral differences. Often, the behavior of optimized networks is sensitive to small perturbations [49]; here, behaviors preserved in the sparsified graph are robust to significant changes.

Laplacian matrices occur in a wide range of physical systems, in many of which, like the spring networks and random walks mentioned earlier, the effect of the spectrum and band gaps is known. In other models, gaps can have significant but less-well-understood effects. We have chosen three nonlinear models to investigate in more detail in order to illustrate the diversity of potential applications for this work. These three systems are each generic, widely studied, and show distinct nontrivial effects of gapped spectra. 
First, we will discuss the Kuramoto model of coupled phase oscillators, typically studied as a model for synchronization. Here, the Laplacian straightforwardly determines the linear behavior near synchronization and has a complex relationship with the nonlinear dynamics. Next, we will present a discretized Swift-Hohenberg type pattern forming system, where controlling the Laplacian spectrum directly controls the steady states of a nonlinear equation. Finally, to diversify our range of physical applications, we will integrate our networks in a quantum mechanical model based on the Gross-Pitaevskii equation. Band gaps in such energy-conserving systems can inhibit transfer of energy between different modes, and we will see how this can tune diffusion of the wave function.

Simulations were performed using a third- or fourthorder Adams-Bashforth linear multistep method with a time step $\Delta t=10^{-4}$. All simulations were written in C++ using ARMADILLO [50].

\section{A. Kuramoto oscillators}

Our first application is the Kuramoto model of coupled oscillators [51,52]. Introduced by Kuramoto in 1975, it has since been studied extensively as a generic setting for interesting synchronization behavior. One of the more surprising discoveries was the existence of chimera states [53], where sets of identical oscillators divide into coexisting synchronized and desynchronized clusters. Commonly considered in networks with identically coupled oscillators, chimeras or chimeralike states can also appear with asymmetric couplings [54]. So far, relatively little attention has been paid to the effect of the spectrum of the coupling network, or, in particular, to what effect a gapped spectrum should have.

Recent experiments coupling Belousov-Zhabotinsky reactions via a computer-controlled projector have shown the emergence of chimeras [14]; we will show this can be achieved in the weak sense of Ref. [55] using our construction with an appropriately gapped spectrum. Here, phases $\theta_{i}$ on the vertices evolve with a natural frequency $\omega$ and a nonlinear coupling defined by the network adjacency matrix:

$$
\frac{d \theta_{i}}{d t}=\omega+\sum_{j=1}^{n} A_{i j} \sin \left(\theta_{j}-\theta_{i}+\alpha\right) .
$$

On a connected graph with $\alpha=0$, there is always a stable attractor $\theta_{i}=\theta_{0}+\omega t$. The rate of convergence to this state within its basin of attraction is controlled by the eigenvalues of the Laplacian $L$ [15].

Both the complete and sparsified exemplar graphs in Fig. 1 have no eigenvalues near zero, so they synchronize much faster than the random graph [Figs. 2(a)-2(c)]. The large gap between the two large clusters of eigenvalues divides the modes into two groups, one synchronizing faster than the other [Figs. 2(d)-2(e)]; moreover, on the complete graph, the localization of the eigenvectors causes staggered synchronization of vertices [Fig. 2(a)].

If $\alpha$ is sufficiently large, the oscillators no longer synchronize at a single frequency. On DBG networks, global coherence gives way to weak chimera states $[55,57]$ where vertices synchronize into two clusters with distinct frequencies [Figs. 2(g)-2(i), Video 1 in Supplemental Material [56]]. For the exactly gapped network with edges of weight $w_{1}=\lambda_{n-1} / n+\left(\lambda_{1}-\lambda_{n-1}\right) /(m+1)$ or $w_{2}=$ $\lambda_{n-1} / n$ described at the end of Sec. III A, where $m=$ $(n / 2)-1$ is the multiplicity of the eigenvalue $\lambda_{1}$, there is a steady state with $\theta_{i}=\theta_{1}$ for $i \leq m+1$ and $\theta_{i}=\theta_{n}$ for $i>m+1$. In this state,

$$
\begin{aligned}
\frac{d}{d t}\left(\theta_{1}-\theta_{n}\right)= & m\left(w_{1}-w_{2}\right) \sin (\alpha) \\
& -2(m+1) w_{2} \sin \left(\theta_{1}-\theta_{n}\right) \cos (\alpha),
\end{aligned}
$$

after some simplification with trigonometric identities. The two phases $\theta_{1}$ and $\theta_{n}$ can synchronize only if there is a solution to

$$
\sin \left(\theta_{1}-\theta_{n}\right)=\frac{m}{2(m+1)}\left(\frac{w_{1}}{w_{2}}-1\right) \tan (\alpha) .
$$

This synchronization is possible if $\alpha$ is small enough that the right-hand side is less than one. If the two groups do not synchronize, and $2(m+1) w_{2}=\lambda_{2}$ is not too large, the second rhs term in Eq. (2) will average to nearly zero giving an approximate mean frequency difference

$$
\left\langle\frac{d}{d t}\left(\theta_{1}-\theta_{n}\right)\right\rangle \approx m\left(w_{1}-w_{2}\right) \sin (\alpha)
$$

which is equal to $-[m /(m+1)]\left(\lambda_{1}-\lambda_{n-1}\right) \sin (\alpha)$ in terms of the eigenvalues. More general cluster synchronization [58,59] could be achieved by adjusting the number and size of the gaps. In contrast, the random graph becomes thoroughly incoherent at comparable values of $\alpha$ [Figs. 2(i) and 2(1)]. The coherence can be quantified by the order parameter $r=\left|\sum_{j} e^{i \theta_{j}}\right|$, which oscillates for the complete and sparsified networks but is near zero for the random graph [Figs. 2(j) and 2(1)], indicating complete disorder.

The result of our construction with a DBG is similar to a standard two-cluster model where chimeras appear [31]. In this model, vertices are divided into two groups of size $n / 2$ with edge weight $-L_{i j}=\mu$ if $i$ and $j$ are in the same cluster and $-L_{i j}=\nu<\mu$ if $i$ and $j$ are in different clusters. In the limit $n \rightarrow \infty$, this system exhibits stable chimera states with one cluster synchronized and the other cluster incoherent. However, for our DBG network, we relax the assumptions that intracluster couplings $\mu$ are identical and stronger than intercluster couplings $\nu$. Perhaps surprisingly, oscillators on the weakly connected side are 

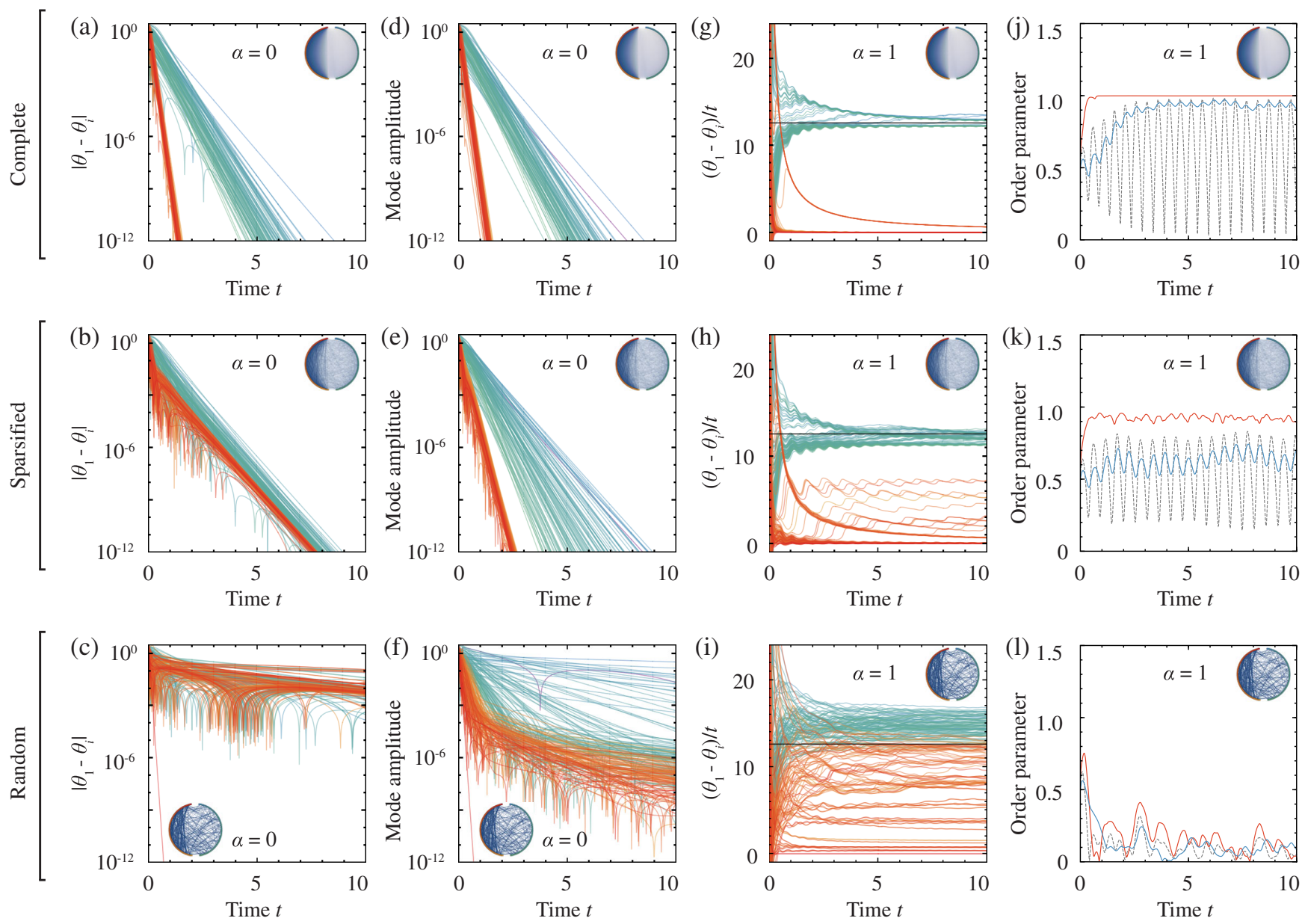

FIG. 2. DBG networks lead to staggered synchronization and chimeras. (a)-(f) In the Kuramoto model with $\alpha=0$, the complete (first row) and sparsified (second row) graphs synchronize much faster than the random graph (third row). For the complete graph the gap affects the rate of synchronization, with highly connected vertices synchronizing faster (a), while on the sparsified graph the gap is only visible in the mode basis (e). (g)-(i) Weak chimera states appear when $\alpha=1$. Both the complete (g) and sparsified (h) graphs have two dominant groups of phase-locked oscillators, with the complete graph more fully synchronized. Dynamics on the random graph (i) are much less coherent. Solid black lines indicate the predicted approximate frequency difference for a network with two distinct eigenvalues, 5 and 20. (j)-(l) Order parameter $r=\left|\sum_{j} e^{i \theta_{j}}\right|$ for the simulations in (g)-(i) for the strongly connected vertices (red), weakly connected vertices (teal), and all vertices (gray). See Video 1 in Supplemental Material [56] for animation.

consistently closer to synchronizing with each other than with strongly connected oscillators, even though the coupling among weakly connected vertices is smaller than the coupling between weakly and strongly connected vertices. Ours are weak chimeras, where we do not require one cluster to be incoherent, but they exist stably on small networks.

The effect appears more related to the gap than to the degeneracy of the eigenvalues. The densely connected side of the sparsified graph is mostly synchronized, even though the eigenvalues above the gap are no longer approximately degenerate. However, the spectrum is not the only determinant of behavior in the Kuramoto model, and potentially there may exist isospectral graphs with qualitatively different dynamics. Compare, for instance, our complete construction with the classic simplest example of a gapped network, a periodic chain with alternating high- and low-weight edges. This does not synchronize in clusters so simply. In fact, on such a graph there are no solutions with two clusters exactly synchronized internally but having distinct long-time average frequencies. Our gapped complete networks can synchronize in distinct clusters because the edge weights are distinct, $w_{1} \neq w_{2}$. Were this not the case, then when the cluster phases coincide with $\theta_{1}=\theta_{n}$, as they must at some point if their frequencies differ, the rhs of Eq. (2) would be zero and the phases would lock. An equivalent argument applies to the alternating chain: all vertices have the same weighted degree, equal to the high weight plus the low weight, which ultimately inhibits clustering.

Greater numbers of clusters can also be considered within this framework. On a network where, for a vertex 

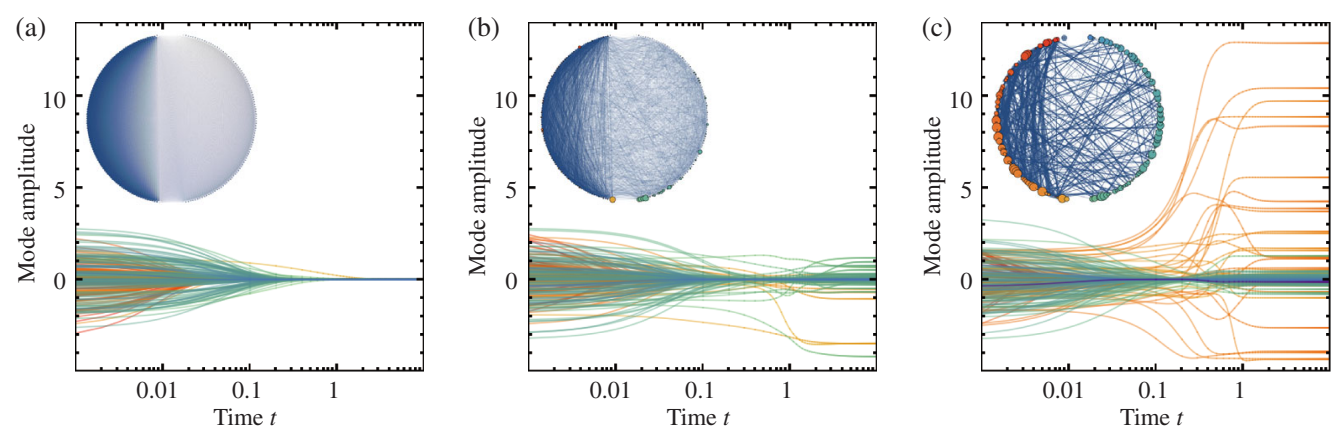

FIG. 3. Generic suppression of pattern formation with a designed discrete band gap. (a) Pattern formation in the Swift-Hohenberg system is completely suppressed by constructing a gap around the range of unstable eigenvalues [Fig. 1(c)]. (b) On a sparsified graph that has a few eigenvalues just within the unstable region, some modes settle at small nonzero values. (c) On the random graph many more eigenvalues are well within the unstable region and the corresponding modes settle at larger amplitudes. The inset graphs show the final steady state on each graph. All simulations used identical initial conditions $u_{i} \sim \mathcal{N}(0,1)$ and parameters $\alpha=90, D_{1}=-20$, $D_{2}=1$. See Video 2 in Supplemental Material [56] for animation.

$i$ in cluster $\beta$, the total weight of edges to vertices in cluster $\gamma$ depends only on $\beta$ and $\gamma$, that is

$$
\sum_{j \in \gamma} A_{i j}=A_{\beta \gamma}
$$

one could work with a reduced Kuramoto model for internally synchronized cluster phases $\left\{\theta_{\beta}\right\}$ reading

$$
\frac{d \theta_{\beta}}{d t}=\omega+\sum_{\gamma} A_{\beta \gamma} \sin \left(\theta_{\gamma}-\theta_{\beta}+\alpha\right)
$$

In this case, if the cluster couplings $A_{\beta \gamma}$ vary sufficiently across clusters then there will exist exact cluster-synchronized solutions. Our DBG networks deviate slightly from these conditions only because of the noise added to the eigenvalues to lift degeneracies. These classes of systems could be realized experimentally in any Kuramoto-type system where the connectivity is controlled. For example, our networks could be input directly into the chemical oscillator system of Ref. [14] where the authors found spiral-wave chimeras.

\section{B. Swift-Hohenberg pattern formation}

As the second application, we study Swift-Hohenberg pattern formation dynamics on a network $[60,61]$. The Swift-Hohenberg equation [60] is a common, generic model of pattern formation, intended to model the selection of patterns with a well-defined scale. It has been applied in areas well beyond the thermally driven convection for which it was developed, ranging from wrinkling of elastic shells [62] to lasers [63]. Though originally defined in a continuous setting, the model can be easily extended to discrete network systems using the analogy between $-\nabla^{2}$ and $L$ [61].

In contexts with a continuous, gapless spectrum of eigenvalues, the standard Swift-Hohenberg parameters define the length scales of the system. With a gap, however, there is an additional interaction between the length scales of pattern formation and the location of the gap. Just as acoustic band gaps can inhibit transmission of sound of particular frequencies [3], gaps in this system should inhibit pattern formation at the corresponding scales.

Consider a scalar field $u_{i}$ on the vertices obeying

$$
\frac{d u_{i}}{d t}=-D_{1} \sum_{j=1}^{n} L_{i j} u_{j}-D_{2} \sum_{j, k=1}^{n} L_{i j} L_{j k} u_{k}-\alpha u_{i}-u_{i}^{3} .
$$

This is the discrete network equivalent to the usual continuous Swift-Hohenberg model $\partial u / \partial t=D_{1} \nabla^{2} u-$ $D_{2} \nabla^{4} u-\alpha u-u^{3}[60,61]$; the extra minus sign in front of the $D_{1}$ term in Eq. (3) arises from the adopted standard sign convention for the discrete graph Laplacian $L$ (Sec. II). The fixed point $u_{i}=0$ of Eq. (3), which exists for any values of the parameters $D_{1}, D_{2}$, and $\alpha$, is linearly stable to perturbations in a Laplacian eigenmode with eigenvalue $\lambda$ if the growth rate $\sigma \equiv-\alpha-D_{1} \lambda-D_{2} \lambda^{2}<0$. With $\alpha$ and $D_{2}$ positive, $\sigma$ is negative for small and large $\lambda$, but choosing $D_{1}<-2 \sqrt{\alpha D_{2}}$ creates a range of unstable $\lambda$ in between. This can drive pattern formation that is eventually stabilized by the nonlinearity. The patterns can only form, however, if $L$ has eigenvalues in the unstable range. Controlling the spectrum of $L$ therefore allows us to completely suppress pattern formation in arbitrarily large systems by placing a gap around the unstable region [Figs. 1(c), 3(a), Video 2 in Supplemental Material [56]]. If we sparsify the network with sufficiently small $\epsilon$, the gap will be preserved and again no patterns will form. Eventually, though, increased sparsification will push some eigenvectors into the edges of the unstable region and bring back partial pattern formation [Fig. 3(b)], which becomes fully developed in the random graph [Fig. 3(c)]. The maximum $\epsilon$ for which patterns will be fully suppressed for given parameter settings can be predicted 
straightforwardly from the expected changes in the eigenvalues, in a similar fashion to the post-sparsification gap size in Fig. 1(d).

Pattern suppression relies only on controlling the available eigenvalues. Our construction, however, has the additional desirable $[16,48]$ feature that the eigenvectors are highly localized. This allows us to control not just whether patterns form, but also which patterns appear. By choosing the eigenvalues of modes corresponding to a set $S$ of vertices to lie inside the unstable range, while leaving all other eigenvalues outside of that range, we can selectively activate only the set $S$. While this only guarantees that the linear part of Eq. (3) selects the desired pattern, because the local nonlinearity only weakly couples different localized modes, the pattern typically survives in the nonlinear regime (Fig. 4, Video 3 in Supplemental Material [56]).

Depending on initial conditions, vertices corresponding to eigenvalues above the unstable range may have small activations, since the activated modes have small but nonzero amplitudes there [Fig. 4(e)]. With random initial conditions, however, positive and negative contributions from activated modes with opposite signs often nearly cancel yielding uniform patterns (Video 3 in Supplemental Material [56], bottom). Uniformity can also be achieved by having no eigenvalues above the unstable range or sufficiently many high eigenvalues that the amplitudes of the activated modes on those vertices is nearly zero. Alternatively, this phenomenon could be used to design more complex patterns with multiple levels of activation.

The conclusion that a band gap can suppress pattern formation does not depend on the discreteness of our system. Experimentally, then, it would be worth investigating both ways to realize a network Swift-Hohenberg model where our networks could be used directly and continuous Swift-Hohenberg systems where a band gap could be introduced.

\section{Gross-Pitaevskii localization}

Having discussed two classical applications to nonconservative systems, we now show how DBGs can control quantum dynamics with conserved energy. In experiments with Bose-Einstein condensates (BECs) in optical lattices [26] researchers often approximate the continuous quantum state with a discrete wave function in the Bose-Hubbard model [64]. Similar Hamiltonians also combining Laplacian-like coupling with local potentials arise for the recently realized fermionic lattice gases [28,29] and connected superconducting waveguides [30].

Assuming the coupling and potentials can be sufficiently well controlled, one can create a network version of the Gross-Pitaevskii model of BEC wave functions. Just as in the Swift-Hohenberg example, we take the
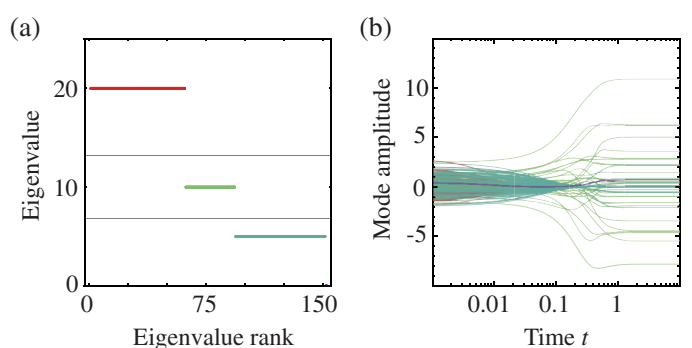

(c) $t=0.00$

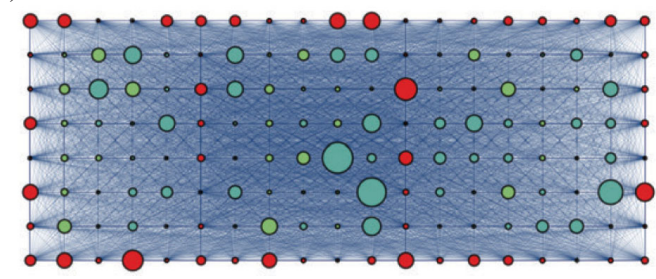

(d) $t=0.07$

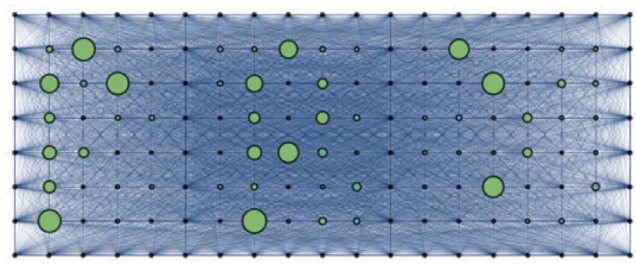

(e) $t=10.0$

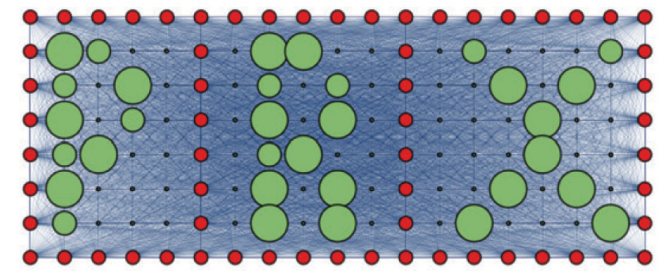

FIG. 4. Controlling pattern formation with a designed discrete band gap (Video 3 in Supplemental Material [56]). (a) Instead of placing a gap in the spectrum around the unstable pattern-forming range, as in Fig. 3, we deliberately place particular eigenvalues in the middle of that range corresponding to eigenvectors localized on a desired pattern. (b) From random initial conditions, the system settles into a state where only the chosen modes have nonnegligible amplitudes. (c)-(e) Time series of pattern evolution on a designed network, with vertices colored according to the stability of the mode localized there as in (a). The size of the vertices indicates $|u|$. (c) The encoded pattern is not obvious from either the designed network or the random initial conditions. (d) By time $t=0.07$ the stable modes have nearly all vanished. (e) The steady state reveals the eigenmode-designed pattern. Because the modes are highly localized, selecting a set of modes to activate is approximately equivalent to selecting a set of vertices to activate. Thus, we can encode an arbitrary pattern as the steady state. Depending on initial conditions, the system may settle into other stable states with slight variations in the vertex activations; the picture typically shows up at least as clearly as it does here and often is more uniform. The parameters $\alpha=90$, $D_{1}=-20$, and $D_{2}=1$ were identical to those in Fig. 3; the tuning parameters to control pattern formation are only the network edge weights. See Video 3 in Supplemental Material [56] for animation and other initial conditions. 
Gross-Pitaevskii equation for a complex wave function $\psi$ and replace the continuous Laplacian $\nabla^{2}$ with its discrete analog $-L$ :

$$
i \frac{d \psi_{j}}{d t}=\sum_{k=1}^{n} L_{j k} \psi_{k}+g\left|\psi_{j}\right|^{2} \psi_{j} .
$$

This discrete nonlinear Schrödinger equation $[65,66]$ can be written $i\left(d \psi_{i} / d t\right)=\partial E / \partial \psi_{i}^{*}$, where the energy $E$ is the sum of the kinetic energy $T=\sum_{i, j} \psi_{i}^{*} L_{i j} \psi_{j}$ and the potential energy $V=\frac{1}{2} g \sum_{j}\left(\psi_{j}^{*} \psi_{j}\right)^{2}$. In the special case of a lattice system, $T$ would include cross-site interaction energies. The potential energy quantifies the localization of $\psi$ : with $g>0$, it is large when the probability $\psi^{*} \psi$ is concentrated at a single vertex and small when $\psi^{*} \psi$ is spread out. Delocalization is limited by the size of the network, as $V \geq g /(2 n)$, but can vary widely even on a finite network. If $\psi$ is initialized at a single vertex $j$, then $V=g / 2$, independent of $j$, while $T=L_{j j}$ equals the degree of $j$.

We find that the interplay of the total energy conservation constraint in such a model with the kinetic energy gap inhibits spreading of the wave function on DBG networks. The effect is reminiscent of Anderson localization [67] and could appear in similar experimental setups [27,68], though the mechanism is distinct. Since energy is conserved, the wave function can delocalize and reduce its potential energy only by converting it to kinetic energy. The rate of potential energy loss, set by $g$, must therefore match the rate of kinetic energy gain, set by the differences in eigenvalues among the modes involved. Suppose the wave function is mostly in a localized mode $j$ with eigenvalue $\lambda_{j}$. Spreading to a higher mode $k$ with $\lambda_{k}-\lambda_{j} \gg g$ would increase kinetic energy by more than it would decrease potential energy, while a weak higher mode $0<\lambda_{k}-\lambda_{j} \ll g$ or a lower mode $\lambda_{k}<\lambda_{j}$ would not increase kinetic energy by enough, if at all. Both are barred by energy conservation. The amplitude in mode $j$ can only be reduced if there are other modes $k$ with $\lambda_{k} \sim \lambda_{j}+g$.

To see this in more detail, suppose we have a wave function comprising two modes, $\psi_{j}=c_{1} v_{j}^{(1)}+c_{2} v_{j}^{(2)}$, with initial complex amplitudes $c_{1}, c_{2}$. Suppose also that these eigenmodes are localized on two different vertices, with $v^{(1)} \approx(-1, \epsilon, \epsilon, \ldots, \epsilon)$ and $v^{(2)} \approx(\epsilon,-1, \epsilon, \ldots, \epsilon)$. The system energy as a function of $c_{1}$ and $c_{2}$ is then

$$
E=\lambda_{1}\left|c_{1}\right|^{2}+\lambda_{2}\left|c_{2}\right|^{2}+\frac{1}{2} g\left[\left|c_{1}\right|^{4}+\left|c_{2}\right|^{4}+O(\epsilon)\right]
$$

If the squared amplitudes change slightly, to $\left|c_{1}\right|^{2}-\delta$ and $\left|c_{2}\right|^{2}+\delta$, the change in energy to leading order in $\delta$ is

$$
\Delta E=\left[\lambda_{2}-\lambda_{1}+g\left(\left|c_{2}\right|^{2}-\left|c_{1}\right|^{2}\right)\right] \delta+O(\epsilon)
$$
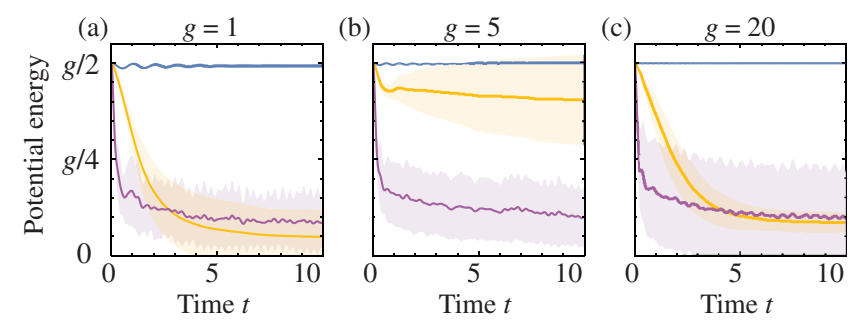

FIG. 5. Localization on a DBG quantum network (Video 4 in Supplemental Material [56]). (a)-(c), When the wave function in the Gross-Pitaevskii model of Eq. (4) is initialized at a weakly connected vertex with low kinetic energy, localization or delocalization (indicated by high or low potential energy, respectively) is controlled by the interplay between the graph spectrum and the rate of potential energy loss $g$. The random graph (purple) always delocalizes, due to its dense spectrum. However, while the sparsified graph (yellow) can delocalize for low $g$ (a) and high $g$ (c), again due to available eigenmodes, intermediate $g$ (b) places the range of allowed modes inside the spectral gap, preventing delocalization. The complete graph (blue) always inhibits spreading due to the extreme localization of its eigenvectors.

Conservation of energy requires $\Delta E=0$, so in order to transfer a noticeable amplitude $\delta \gg \epsilon$ from the first mode to the second we must have $\lambda_{2}-\lambda_{1}+g\left(\left|c_{2}\right|^{2}-\left|c_{1}\right|^{2}\right) \approx 0$. In the cases considered in Fig. 5, where $\left|c_{1}\right| \approx 1$ and $\left|c_{2}\right| \approx 0$, this reduces to $\lambda_{2}-\lambda_{1} \approx g$. Thus, on a network with a spectral gap, the localization of $\psi$ can depend nontrivially on the interplay between $g$ and the spectrum.

Initializing $\psi$ at a weakly connected vertex brings out this interplay as $g$ is varied (Video 4 in Supplemental Material [56]). The initial state, with high potential energy and low kinetic energy, is localized on modes with an eigenvalue below the spectral gap. On the sparsified network, a low value of $g$ makes nearby modes below the gap accessible for delocalization, causing the wave function to spread [Fig. 5(a)]. However, increasing $g$ pushes the region where transfer is possible inside the spectral gap, inhibiting the spread of the wave function on the sparsified network [Fig. 5(b)]. A further increase of $g$ once again enables delocalization as the modes above the gap become accessible for energy transfer [Fig. 5(c)]. In contrast, the dense spectrum of the random graph means delocalization occurs in all three instances (Fig. 5). Interestingly, the complete DBG network appears to remain localized for all values of $g$ in our simulations (Fig. 5); this is likely due to the strong localization and near-zero overlap of the eigenmodes.

Experimentally, this could be realized either with BECs in appropriately tuned optical potentials or as a physical network of waveguides. Building the large complete networks we introduced is beyond current experimental techniques but may be possible with future advancements. For the short term, we will show in the next section how extending smaller, low-connectivity networks periodically can lead to more practical networks with approximately the same spectral properties. 


\section{BAND STRUCTURE IN PERIODIC NETWORKS}

High connectivity can make it difficult to build experimental versions of complex networks. This motivated our study of spectral sparsification, as the sparsified graphs should be easier to realize in an experiment. An alternative approach to making practically usable networks is to create a periodic crystal where we control the behavior of the unit cell. Keeping the unit cell small will reduce the connectivity and simplify fabrication, while the periodic structure will enable building on a larger scale. The key question is then, can the spectrum of the full system can be controlled by tuning only the unit cell? In what follows, we show that it can.

We can construct infinite periodic networks in a standard way from any base network $G$ by tiling periodically and rewiring edges [Figs. 6(a) and 6(d)]. Starting from the original vertex set $\{j\}$ for $1 \leq j \leq n$ and edge weights $-L_{j k}$, we make an infinite string $G^{\infty}$ of copies of $G$ with vertices indexed by $j$, the label in $G$, and $c \in \mathbb{Z}$, the unit cell. This will give a new, infinite Laplacian $L^{\infty}$. For the edges that will not be rewired, we set $L_{j c, k c}^{\infty}=L_{j k}$ for all $c$. Doing this for all edges would leave the copies of $G$ disconnected. To connect them, we choose a subset of edges $\{(j, k)\}$ and rewire them to cross between unit cells; e.g., if $(j, k)$ is an edge to be rewired to have $k$ in a unit cell to the left of $j$ we can set $L_{j c, k(c-1)}^{\infty}=L_{j k}$ for all $c$ and symmetrically set $L_{k(c-1), j c}^{\infty}=L_{k j}$. The remainder of the entries of $L^{\infty}$ are set to zero.

Since $L^{\infty}$ is periodic, Bloch's theorem allows us to write the eigenvectors as

$$
U_{j c}^{\infty}(q)=e^{i q c} \tilde{U}_{j}(q),
$$

where $q$ is a wave number in the first Brillouin zone $-\pi<q<\pi$. The $\tilde{U}$ then satisfy

$$
\lambda(q) e^{i q c} \tilde{U}_{j}(q)=\sum_{k, d} L_{j c, k d}^{\infty} e^{i q d} \tilde{U}_{k}(q),
$$

which reduces to a new eigenvalue equation for a matrix of size $n$ :

$$
\lambda(q) \tilde{U}(q)=\tilde{L}(q) \tilde{U}(q),
$$

where the matrix elements of $\tilde{L}(q)$ are the same as those of $L$ for edges within a single unit cell and differ by a factor $e^{i q(c-d)}$ for edges that cross between unit cells $c$ and $d$.

Using these transformations, which are standard in the study of lattice systems [17], we can find the continuous spectra of periodic tilings of our designed networks. Even without any optimization of which edges to rewire, the spectral characteristics persist in the infinite (a)

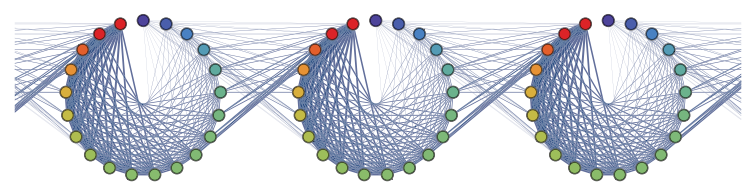

(d)

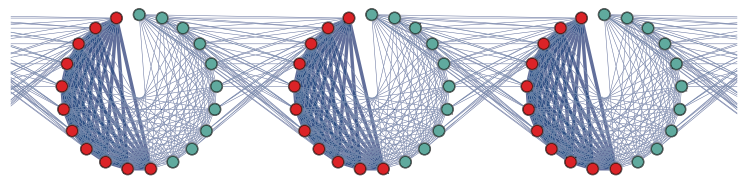

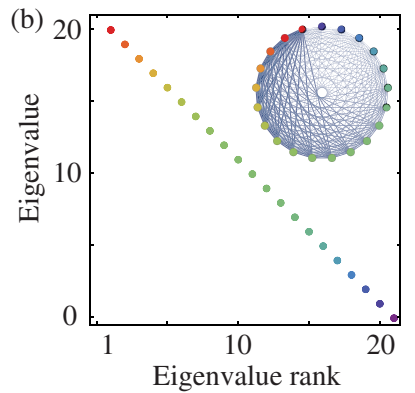
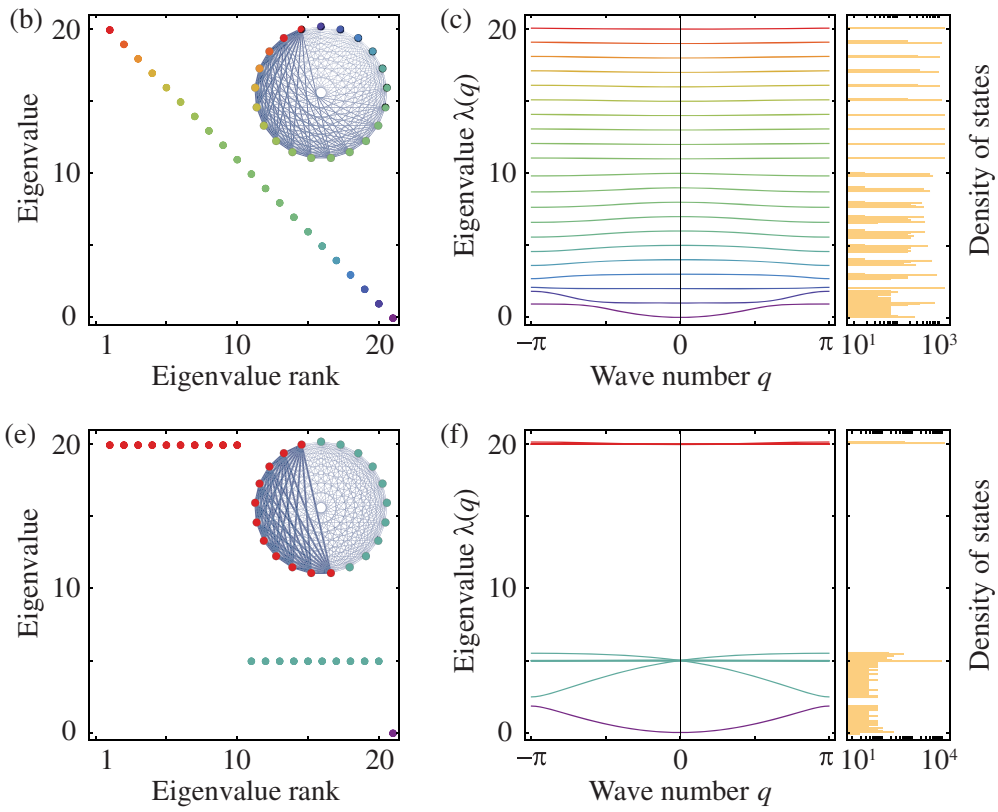

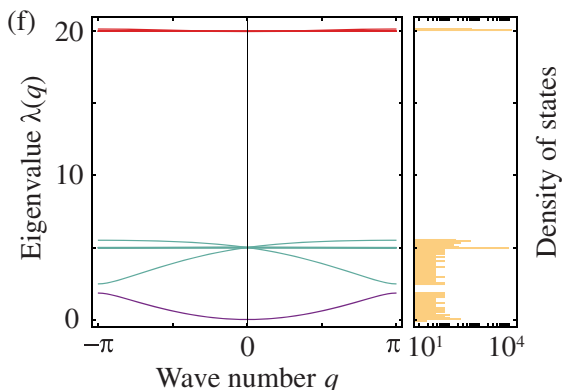

FIG. 6. Designed spectra on a discrete network are preserved when extended periodically in one dimension. (a) We extend a finite network to an infinite one by rewiring a subset of the edges to cross between adjacent copies of the original network. Here, we take the network with the spectrum in (b) and rewire the edge between vertices $j$ and $k$ if $|k-j|>n / 2$. This rewires roughly one quarter of the edges. (b) One unit cell in (a) would have a discrete spectrum with $\lambda_{j}=21-j$. (c) Most of the eigenvalue bands do not change significantly with $q$, so the density of states consists of 21 sharp peaks with low- or zero-density regions between. (d) The same construction as in (a) can be repeated for any spectrum; this is the result for a gapped network. (e) One unit cell in (d) would have a gapped spectrum, with 10 eigenvalues equal to 20 and 10 equal to 5, in addition to the always-present zero eigenvalue. (f) Again, most of the eigenvalue bands are roughly constant, even though the eigenvectors do depend strongly on $q$. The gap in the middle of the spectrum is nearly perfectly preserved; a small gap remains between the bottom two bands. Note the log scale on both density of states plots. 
system. If we rewire all edges with $|j-k|>n / 2$, for example a spectrum of equally spaced eigenvalues leads to a density of states with corresponding equally spaced large spikes [Figs. 6(a)-6(c)], while a discrete band gap is almost entirely preserved [Figs. 6(d)-6(f)]. In both cases, only the bottom few bands vary significantly with $q$. Note that because we moved edges incident to the first vertex, all of the eigenvectors do change and are not localized for nonzero $q$.

\section{CONCLUSIONS}

Controlling dynamics on a network typically requires detailed understanding of its spectral properties. Here, we have reversed the conventional approach by starting from a desired spectrum and providing a mathematically rigorous construction of a matching network. This enables us to induce chimera states, suppress or fine-tune pattern formation, and control wave-function localization [69] using suitably designed gapped spectra. We introduce our three applications only to showcase a few possibilities and we expect the construction to be useful in many other contexts. Still, these three already provide opportunities for experimental realizations, including computer-coupled chemical oscillators [14], cold atomic systems with precisely specified optical potentials [26-29,64], or etched superconducting waveguide resonators [30]. For metamaterials that can be approximated as spring networks, designing the Laplacian would determine the transmission properties and allow selective acoustic damping. Diffusive transport networks designed with these principles could have mixing times controlled independently for different initial conditions. Any of these models can also be naturally extended to periodic systems, where the spectral properties are preserved well without any further optimization. Judicious use of designed networks could aid both discovery, where fine control allows more precise measurement and understanding, and application, where optimizing transmission spectra [3], transport and localization of electrons $[12,67]$, or the timing of chemical reactions [70] can have immediate consequences. Furthermore, chimera networks promise custom-designed frequency filters and control of secondary transition and resonance phenomena [71] through tailored multimodal fluctuation spectra.

Our method, which starts from global properties, complements traditional approaches using small-scale local rules to build and analyze networks [35,72-74]. In the future, the above results may also prove useful as a standard of comparison for other networks. Contrasting the dynamics on an important class of networks with the dynamics on networks designed to have identical spectra can help identify the important features of that class. Moreover, as dynamics are often related to matrices other than the Laplacian [75], it will be interesting to investigate control of their spectra for weighted networks as well. Although our construction works optimally with fully connected graphs, one can expect that improved sparsification algorithms together with recent progress in 3D printing and lithography $[18,24]$ may soon lead to physically realizable networks with arbitrary gaps; since any graph can be embedded in 3D [76], the framework introduced here lays a conceptual foundation for the targeted design of complex nonperiodic metamaterials with desired spectral properties.

\section{ACKNOWLEDGMENTS}

The authors would like to thank Jon Kelner and Philippe Rigollet for helpful discussions. This work was supported by Trinity College, Cambridge (F. G. W.), an Edmund F. Kelly Research Award (J.D.), the MIT Solomon Buchsbaum Research Fund (J.D.), and a Complex Systems Scholar Award of the James S. McDonnell Foundation (J. D.).

\section{APPENDIX A: EDGE WEIGHTS WITH A GAP}

Suppose we have eigenvalues $\lambda_{1}$ with multiplicity $m$ and $\lambda_{n-1}<\lambda_{1}$ with multiplicity $n-m-1$. Then, if $i<j \leq$ $m+1$,

$$
\begin{aligned}
L_{i j} & =-\frac{\lambda_{1}}{j}+\sum_{k=j}^{m} \frac{\lambda_{1}}{k(k+1)}+\sum_{k=m+1}^{n-1} \frac{\lambda_{n-1}}{k(k+1)} \\
& =-\frac{\lambda_{1}}{m+1}+\lambda_{n-1}\left(\frac{1}{m+1}-\frac{1}{n}\right) .
\end{aligned}
$$

Else, if $i<j$ and $j>m+1$,

$$
L_{i j}=\lambda_{n-1}\left[-\frac{1}{j}+\sum_{k=j}^{n-1} \frac{1}{k(k+1)}\right]=-\frac{\lambda_{n-1}}{n} .
$$

There are two types of edges: edges with both end points in the first $m+1$ vertices have weight $\lambda_{n-1} / n+\left(\lambda_{1}-\lambda_{n-1}\right) /$ $(m+1)$, while other edges have weight $\lambda_{n-1} / n$.

\section{APPENDIX B: POSITIVITY OF EDGE WEIGHTS}

The elements of the designed $L$ above the diagonal, $L_{i j}$ for $i<j$, are given by

$$
\begin{aligned}
L_{i j} & =\sum_{k=1}^{n-1} \lambda_{k} u_{i}^{(k)} u_{j}^{(k)} \\
& =\lambda_{j-1} u_{i}^{(j-1)} u_{j}^{(j-1)}+\sum_{k=j}^{n-1} \lambda_{k} u_{i}^{(k)} u_{j}^{(k)} \\
& \leq \lambda_{j-1}\left(-\frac{1}{j}+\sum_{k=j}^{n-1} \frac{1}{k(k+1)}\right) \\
& =\lambda_{j-1}\left(-\frac{1}{j}+\frac{1}{j}-\frac{1}{n}\right) \\
& =-\frac{\lambda_{j-1}}{n} \leq 0 .
\end{aligned}
$$


From the second to third lines we use the definition of the eigenvectors in Eq. (1); the sum $\sum_{k=j}^{n-1} 1 /[k(k+1)]=$ $(1 / j)-(1 / n)$ in the third line can be computed as a telescoping sum of partial fractions. $L$ is symmetric, so the elements below the diagonal must also be nonpositive. This proves that the edge weights of the constructed graph are non-negative.

\section{APPENDIX C: RANDOM MATCHED-DEGREE GRAPHS}

Given a weighted graph $G$, we can construct a random simple graph $\tilde{G}$ with the same vertex degrees as $G$ in a way analogous to known methods for sampling random regular graphs [77]. Let $w(e)$ denote the weight of edge $e$ and $d(v)$ denote the weighted degree of vertex $v$. Begin with a disconnected graph with a loop of weight $d(v) / 2$ at each vertex $v$; this has the same degrees as $G$ but is not simple. Repeat the following steps until there are no loops:

(1) Pick a loop $l=(u, u)$ and another edge $e=(v, w)$ at random, with $v \neq u \neq w$.

(2) (a) If $w(l)>w(e)$, remove $e$ and add $e^{\prime}=(u, v)$ and $e^{\prime \prime}=(u, w)$ with weight $w(e)$. Subtract $w(e)$ from the weight of $l$.

(b) Else, remove $l$ and add $e^{\prime}=(u, v)$ and $e^{\prime \prime}=$ $(u, w)$ with weight $w(l)$. Subtract $w(l)$ from the weight of $e$.

Once there are no more loops, merge all sets of edges between the same pair of vertices into one edge with the same total weight. Since the degree of each vertex is preserved at each step, the final graph has the same degrees as $G$. In the examples considered here, the algorithm terminates quickly.

[1] M.Z. Hasan and C. L. Kane, Colloquium: Topological Insulators, Rev. Mod. Phys. 82, 3045 (2010).

[2] B. Bradlyn, L. Elcoro, J. Cano, M. G. Vergniory, Z. Wang, C. Felser, M. I. Aroyo, and B. A. Bernevig, Topological Quantum Chemistry, Nature (London) 547, 298 (2017).

[3] O. Sigmund and J.S. Jensen, Systematic Design of Phononic Band-Gap Materials and Structures by Topology Optimization, Phil. Trans. R. Soc. A 361, 1001 (2003).

[4] J. Bardeen, L. N. Cooper, and J. R. Schrieffer, Theory of Superconductivity, Phys. Rev. 108, 1175 (1957).

[5] P. Wang, Y. Zheng, M. C. Fernandes, Y. Sun, K. Xu, S. Sun, S. H. Kang, V. Tournat, and K. Bertoldi, Harnessing Geometric Frustration to Form Band Gaps in Acoustic Channel Lattices, Phys. Rev. Lett. 118, 084302 (2017).

[6] A. Souslov, B. C. van Zuiden, D. Bartolo, and V. Vitelli, Topological Sound in Active-Liquid Metamaterials, Nat. Phys. 13, 1091 (2017).

[7] B. Sonnenschein, M. A. Zaks, A. B. Neiman, and L. Schimansky-Geier, Excitable Elements Controlled by Noise and Network Structure, Eur. Phys. J. Spec. Top. 222, 2517 (2013).
[8] G. Bianconi, Interdisciplinary and Physics Challenges of Network Theory, Europhys. Lett. 111, 56001 (2015).

[9] H. Ronellenfitsch and E. Katifori, Global Optimization, Local Adaptation, and the Role of Growth in Distribution Networks, Phys. Rev. Lett. 117, 138301 (2016).

[10] A. M. Tayar, E. Karzbrun, V. Noireaux, and R. H. Bar-Ziv, Propagating Gene Expression Fronts in a One-Dimensional Coupled System of Artificial Cells, Nat. Phys. 11, 1037 (2015).

[11] D. Brockmann, L. Hufnagel, and T. Geisel, The Scaling Laws of Human Travel, Nature (London) 439, 462 (2006).

[12] J. E. Cohen and P. Horowitz, Paradoxical Behaviour of Mechanical and Electrical Networks, Nature (London) 352, 699 (1991).

[13] S.-H. Yook, H. Jeong, and A.-L. Barabási, Modeling the Internet's Large-Scale Topology, Proc. Natl. Acad. Sci. U. S. A. 99, 13382 (2002).

[14] J. F. Totz, J. Rode, M. R. Tinsley, K. Showalter, and H. Engel, Spiral Wave Chimera States in Large Populations of Coupled Chemical Oscillators, Nat. Phys. 14, 282 (2018).

[15] P. N. McGraw and M. Menzinger, Laplacian Spectra as a Diagnostic Tool for Network Structure and Dynamics, Phys. Rev. E 77, 031102 (2008).

[16] H. Nakao and A. S. Mikhailov, Turing Patterns in NetworkOrganized Activator-Inhibitor Systems, Nat. Phys. 6, 544 (2010).

[17] T. C. Lubensky, C. L. Kane, X. Mao, A. Souslov, and K. Sun, Phonons and Elasticity in Critically Coordinated Lattices, Rep. Prog. Phys. 78, 073901 (2015).

[18] W. Man, M. Florescu, K. Matsuyama, P. Yadak, G. Nahal, S. Hashemizad, E. Williamson, P. Steinhardt, S. Torquato, and P. Chaikin, Photonic Band Gap in Isotropic Hyperuniform Disordered Solids with Low Dielectric Contrast, Opt. Express 21, 19972 (2013).

[19] M. Y. Han, B. Özyilmaz, Y. Zhang, and P. Kim, Energy Band-Gap Engineering of Graphene Nanoribbons, Phys. Rev. Lett. 98, 206805 (2007).

[20] A. S. Gladman, E. A. Matsumoto, R. G. Nuzzo, L. Mahadevan, and J.A. Lewis, Biomimetic 4D Printing, Nat. Mater. 15, 413 (2016).

[21] K. Wang, Y.-H. Chang, Y. Chen, C. Zhang, and B. Wang, Designable Dual-Material Auxetic Metamaterials Using Three-Dimensional Printing, Mater. Des. 67, 159 (2015).

[22] N. Bhattacharjee, A. Urrios, S. Kang, and A. Folch, The Upcoming 3D-Printing Revolution in Microfluidics, Lab Chip 16, 1720 (2016).

[23] L. Huang, R. Jiang, J. Wu, J. Song, H. Bai, B. Li, Q. Zhao, and T. Xie, Ultrafast Digital Printing toward 4D Shape Changing Materials, Adv. Mater. 29, 1605390 (2017).

[24] T. Bückmann, N. Stenger, M. Kadic, J. Kaschke, A. Frölich, T. Kennerknecht, C. Eberl, M. Thiel, and M. Wegener, Tailored 3D Mechanical Metamaterials Made by Dip-In Direct-Laser-Writing Optical Lithography, Adv. Mater. 24, 2710 (2012).

[25] A. E. Motter, Networkcontrology, Chaos 25, 097621 (2015).

[26] M. Greiner, O. Mandel, T. Esslinger, T. W. Hänsch, and I. Bloch, Quantum Phase Transition from a Superfluid to a Mott Insulator in a Gas of Ultracold Atoms, Nature (London) 415, 39 (2002). 
[27] J. Billy, V. Josse, Z. Zuo, A. Bernard, B. Hambrecht, P. Lugan, D. Clément, L. Sanchez-Palencia, P. Bouyer, and A. Aspect, Direct Observation of Anderson Localization of Matter Waves in a Controlled Disorder, Nature (London) 453, 891 (2008).

[28] L. W. Cheuk, M. A. Nichols, M. Okan, T. Gersdorf, V. V. Ramasesh, W. S. Bakr, T. Lompe, and M. W. Zwierlein, Quantum-Gas Microscope for Fermionic Atoms, Phys. Rev. Lett. 114, 193001 (2015).

[29] M. A. Nichols, L. W. Cheuk, M. Okan, T. R. Hartke, E. Mendez, T. Senthil, E. Khatami, H. Zhang, and M. W. Zwierlein, Spin Transport in a Mott Insulator of Ultracold Fermions, arXiv:1802.10018.

[30] A. J. Kollár, M. Fitzpatrick, and A. A. Houck, Hyperbolic Lattices in Circuit Quantum Electrodynamics, arXiv:1802 .09549 .

[31] M. J. Panaggio and D. M. Abrams, Chimera States: Coexistence of Coherence and Incoherence in Networks of Coupled Oscillators, Nonlinearity 28, R67 (2015).

[32] E. A. Martens, S. Thutupalli, A. Fourriére, and O. Hallatschek, Chimera States in Mechanical Oscillator Networks, Proc. Natl. Acad. Sci. U. S. A. 110, 10563 (2013).

[33] D. A. Spielman and N. Srivastava, Graph Sparsification by Effective Resistances, SIAM J. Comput. 40, 1913 (2011).

[34] J. A. Kelner and A. Levin, Spectral Sparsification in the Semi-Streaming Setting, Theory Comput. Syst. 53, 243 (2013).

[35] A.-L. Barabasi and R. Albert, Emergence of Scaling in Random Networks, Science 286, 509 (1999).

[36] A. Arenas, A. Díaz-Guilera, and C. J. Pérez-Vicente, Synchronization Reveals Topological Scales in Complex Networks, Phys. Rev. Lett. 96, 114102 (2006).

[37] T. P. Peixoto, Eigenvalue Spectra of Modular Networks, Phys. Rev. Lett. 111, 098701 (2013).

[38] A. Amir, Y. Oreg, and Y. Imry, Localization, Anomalous Diffusion, and Slow Relaxations: A Random Distance Matrix Approach, Phys. Rev. Lett. 105, 070601 (2010).

[39] G. Huiskamp, Difference Formulas for the Surface Laplacian on a Triangulated Surface, J. Comput. Phys. 95, 477 (1991).

[40] R. A. Brualdi and S. Mellendorf, Regions in the Complex Plane Containing the Eigenvalues of a Matrix, Am. Math. Mon. 101, 975 (1994).

[41] M. Ipsen and A. S. Mikhailov, Evolutionary Reconstruction of Networks, Phys. Rev. E 66, 046109 (2002).

[42] F. Comellas and J. Diaz-Lopez, Spectral Reconstruction of Complex Networks, Physica (Amsterdam) 387A, 6436 (2008).

[43] D. Cvetkovič, Spectral Recognition of Graphs, Yugoslav Journal of operations research 22, 145 (2012).

[44] B. D. McKay, On the Spectral Characterisation of Trees, Ars Combinatoria 3, 219 (1977).

[45] L. Halbeisen and N. Hungerbühler, Reconstruction of Weighted Graphs by Their Spectrum, Eur. J. Combinatorics 21, 641 (2000).

[46] E. R. Van Dam and W. H. Haemers, Which Graphs are Determined by Their Spectrum?, Linear Algebra Appl. 373, 241 (2003).

[47] A. E. Motter, Bounding Network Spectra for Network Design, New J. Phys. 9, 182 (2007).
[48] P. Pradhan, A. Yadav, S. K. Dwivedi, and S. Jalan, Optimized Evolution of Networks for Principal Eigenvector Localization, Phys. Rev. E 96, 022312 (2017).

[49] T. Nishikawa, J. Sun, and A. E. Motter, Sensitive Dependence of Optimal Network Dynamics on Network Structure, Phys. Rev. X 7, 041044 (2017).

[50] C. Sanderson and R. Curtin, ARMADILLO: A Template-Based C++ Library for Linear Algebra, J. Open Source Software 1, 26 (2016).

[51] Y. Kuramoto, Self-Entrainment of a Population of Coupled Non-Linear Oscillators, Int. Symp. Math. Prob. Theor. Phys. 39, 420 (1975).

[52] S. H. Strogatz, From Kuramoto to Crawford: Exploring the Onset of Synchronization in Populations of Coupled Oscillators, Physica (Amsterdam) 143D, 1 (2000).

[53] Y. Kuramoto and D. Battogtokh, Coexistence of Coherence and Incoherence in Nonlocally Coupled Phase Oscillators, Nonlinear Phenom. Complex Syst. 5, 380 (2002).

[54] X. Jiang and D. M. Abrams, Symmetry-Broken States on Networks of Coupled Oscillators, Phys. Rev. E 93, 052202 (2016).

[55] P. Ashwin and O. Burylko, Weak Chimeras in Minimal Networks of Coupled Phase Oscillators, Chaos 25, 013106 (2015).

[56] See Supplemental Material at http://link.aps.org/supplemental/ 10.1103/PhysRevX.8.041043 for Videos 1-4.

[57] C. Bick, M. Sebek, and I. Z. Kiss, Robust Weak Chimeras in Oscillator Networks with Delayed Linear and Quadratic Interactions, Phys. Rev. Lett. 119, 168301 (2017).

[58] Y. S. Cho, T. Nishikawa, and A. E. Motter, Stable Chimeras and Independently Synchronizable Clusters, Phys. Rev. Lett. 119, 084101 (2017).

[59] L. M. Pecora, F. Sorrentino, A. M. Hagerstrom, T. E. Murphy, and R. Roy, Cluster Synchronization and Isolated Desynchronization in Complex Networks with Symmetries, Nat. Commun. 5, 4079 (2014).

[60] J. Swift and P. C. Hohenberg, Hydrodynamic Fluctuations at the Convective Instability, Phys. Rev. A 15, 319 (1977).

[61] C. Nicolaides, R. Juanes, and L. Cueto-Felgueroso, SelfOrganization of Network Dynamics into Local Quantized States, Sci. Rep. 6, 21360 (2016).

[62] N. Stoop, R. Lagrange, D. Terwagne, P. M. Reis, and J. Dunkel, Curvature-Induced Symmetry Breaking Determines Elastic Surface Patterns, Nat. Mater. 14, 337 (2015).

[63] J. Lega, J. V. Moloney, and A. C. Newell, Swift-Hohenberg Equation for Lasers, Phys. Rev. Lett. 73, 2978 (1994).

[64] I. Brunelli, G. Giusiano, F. P. Mancini, P. Sodano, and A. Trombettoni, Topology-Induced Spatial Bose-Einstein Condensation for Bosons on Star-Shaped Optical Networks, J. Phys. B 37, S275 (2004).

[65] O. Bang, J. J. Rasmussen, and P. L. Christiansen, Subcritical Localization in the Discrete Nonlinear Schrodinger Equation with Arbitrary Power Nonlinearity, Nonlinearity 7, 205 (1994).

[66] D. E. Pelinovsky, D. A. Zezyulin, and V. V. Konotop, Nonlinear Modes in a Generalized PT-Symmetric Discrete Nonlinear Schrödinger Equation, J. Phys. A 47, 085204 (2014).

[67] P. W. Anderson, Absence of Diffusion in Certain Random Lattices, Phys. Rev. 109, 1492 (1958). 
[68] G. Roati, C. D'Errico, L. Fallani, M. Fattori, C. Fort, M. Zaccanti, G. Modugno, M. Modugno, and M. Inguscio, Anderson Localization of a Non-Interacting Bose-Einstein Condensate, Nature (London) 453, 895 (2008).

[69] A. Amir, J. J. Krich, V. Vitelli, Y. Oreg, and Y. Imry, Emergent Percolation Length and Localization in Random Elastic Networks, Phys. Rev. X 3, 021017 (2013).

[70] A. M. Turing, The Chemical Basis of Morphogenesis, Phil. Trans. R. Soc. B 237, 37 (1952).

[71] L. Gammaitoni, P. Hänggi, P. Jung, and F. Marchesoni, Stochastic Resonance, Rev. Mod. Phys. 70, 223 (1998).

[72] G. Bianconi and A.-L. Barabási, Competition and Multiscaling in Evolving Networks, Europhys. Lett. 54, 436 (2001).
[73] T. Lorimer, F. Gomez, and R. Stoop, Two Universal Physical Principles Shape the Power-Law Statistics of Real-World Networks, Sci. Rep. 5, 12353 (2015).

[74] R. Milo, S. Shen-Orr, S. Itzkovitz, N. Kashtan, D. Chklovskii, and U. Alon, Network Motifs: Simple Building Blocks of Complex Networks, Science 298, 824 (2002).

[75] G. Yan, G. Tsekenis, B. Barzel, J.-J. Slotine, Y.-Y. Liu, and A.-L. Barabási, Spectrum of Controlling and Observing Complex Networks, Nat. Phys. 11, 779 (2015).

[76] R. F Cohen, P. Eades, T. Lin, and F. Ruskey, ThreeDimensional Graph Drawing, Algorithmica 17, 199 (1997).

[77] B. McKay and N. Wormald, Uniform Generation of Random Regular Graphs, J. Algorithms 11, 52 (1990). 